\title{
Las relaciones públicas en las campañas electorales
}

Recibido: 16 de noviembre de 2018

Aceptado: 29 de enero de 2019

Publicado: 24 de junio de 2019
Andrés Valdez Zepeda

azepeda@cucea.udg.mx

Universidad de Guadalajara (México)

Resumen: El escrito hace una descripción del papel estratégico que juegan las relaciones públicas en las campañas electorales. Se inicia con la conceptualización de las relaciones públicas y su relación con la política (en lo general) y con la política electoral (en lo particular). Se argumenta que el impulso de una campaña electoral implica un ejercicio de relaciones públicas con el objetivo de conocer a los electores y de diseñar diferentes estrategias para conseguir su voto y así evitar que los adversarios logren arribar a posiciones de representación pública. Se concluye que el éxito o fracaso de una campaña electoral dependerá de la capacidad que se tenga como individuos o como organizaciones para lograr un manejo creativo, oportuno e inteligente de las relaciones públicas.

Palabras clave: Relaciones públicas, campañas electorales, democracia y gestión estratégica.

Abstract: The essay makes a description of the strategical role the play public relations during electoral campaigns. In the beginning, the essay conceptualizes public relations and its connection with political campaigns. It is argued that make an electoral campaign imply a public relation activity with the objective of knowing the voters and make up different political strategies to get their votes and, consequently, avoid the opposition can win the political power. It is concluded that the successful in a political campaigns depends on the creative, intelligent and innovative use of public relations.

Key words: Public relations, electoral campaigns, democracy and strategic management. 


\section{Introducción}

La política es una actividad humana que implica relaciones e interrelaciones entre personas y/o grupos de personas, quienes se asocian para diferentes fines, como puede ser, por ejemplo, apoyar proyectos comunes, conservar o acceder a posiciones de poder, lograr un objetivo común o incluso, para confrontar y derrotar a quienes consideran sus adversarios. Es decir, de cierta manera la política es un "deporte de contacto", que implica la relación de un individuo con otro o con un grupo de individuos para lograr un determinado fin. De hecho, se puede asegurar que no puede haber política sin relación, porque la política en sí mismo implica una relación.

Dentro de la política, se encuentra la política electoral, la cual puede ser definida como las acciones de relación e inter-relación, comunicación y organización realizadas por una o más personas o grupos de personas con el fin de persuadir a otros para construir mayorías electorales y así poder acceder a un puesto de representación pública (Godínez y Cornejo, 2017). Como parte de la política electoral en sistemas de cuño democrático, se impulsan las campañas electorales, mismas que son definidas como las acciones de relación, interrelación y comunicación política que se realizan por personas a nombre de ellas, de segundas personas o de sus organizaciones con el fin de obtener el voto de los electores con el fin de conservar o acceder a un puesto de representación (Valdez, 2018).

Estas campañas electorales, se pueden impulsar en organizaciones o entes de carácter privado como las empresas, los sindicatos o las organizaciones estudiantiles, entre otras o de carácter público, como pueden ser los diferentes poderes públicos de un Estado-nación (ejecutivo, legislativo o judicial) o los diferentes niveles de gobierno (municipal, estatal y federal), entre otros. De esta manera, las relaciones públicas están presentes como medio y herramienta importante dentro de las campañas electorales, cumpliendo un papel relevante para alcanzar su objetivo central que es conservar o acceder a los puestos de poder político o cargos de representación.

Las relaciones públicas se encuentran presentes en las acciones referentes a la investigación de la opinión pública y en las acciones para incidir en crear una opinión pública favorable para el candidato y el partido que los postula. De hecho, una parte importante de las actividades de un publirrelacionista es la de investigar la opinión pública para saber sobre los problemas, necesidades, preferencias, simpatías, deseos, gustos y emociones de los votantes. A partir del diagnóstico, se diseña una estrategia publicitaria, generalmente, segmentada por sectores sociales para tratar de incidir en el comportamiento de los electores.

Otra labor muy importante de las relaciones públicas durante las campañas electorales también es la publicidad de los proyectos políticos y de los candidatos a un puesto de elección popular a través de sendas campañas en medios de comunicación (radio, prensa y televisión) y en las nuevas redes sociales que usan la internet y las nuevas tecnologías de la comunicación y la información. La idea central es darle visibilidad social al candidato y a sus propuestas, acercándolo con los electores, para que éstos lo conozcan y, en su momento, puedan emitir el voto a su favor. 
Fomentar relaciones con liderazgos y con diferentes sectores sociales es otra de las labores importantes de un publi-relacionista. De hecho, esta es una labor central, ya que un candidato debe mantener relaciones afectivas con una base amplia de electores, quienes no solo deben votar por él, sino también ayudarle a hacer campaña entre sus conocidos. Es decir, a través de una buena estrategia de relaciones públicas, un candidato a un puesto de elección popular puede lograr que los electores hagan campaña por él, tratando de convertir a la campaña en un movimiento social que favorezca su candidatura. Un publi-relacionista también impulsa, durante la campaña electoral, diferentes acciones de comunicación con las audiencias a través de campañas publicitarias en medios de comunicación, también a través de las redes sociales o por actos y eventos de contacto directo con el elector.

Tener buenas relaciones con los medios, para que estos cubran los eventos del candidato y de la campaña electoral, es otra de las funciones centrales que realiza el publirelacionista. La idea es que el candidato esté presente, de manera frecuente, en los medios de comunicación en entrevistas, ruedas de prensa y diferentes actos que lo ayuden a ser conocido, posicionarse y diferenciarse respecto de la competencia.

Gestionar las crisis que se presenten durante la campaña electoral, protagonizada ya sea por el candidato, algún miembro distinguido del partido político que lo postula o del equipo de campaña también es una función central de los publi-relacionistas, tratando de resolver de inmediato la crisis o evitando que las consecuencias políticas sean mayores.

En este escrito, se hablará sobre el papel estratégico que juegan las relaciones públicas durante las campañas electorales. El objetivo del escrito es describir y analizar el papel que juegan las relaciones públicas durante las campañas electorales en sistemas de cuño democrático, describiendo aspectos generales y particulares de su uso.

La metodología utilizada en el escrito es de carácter descriptiva, dando cuenta de las áreas y acciones donde las relaciones públicas se encuentran presentes durante las campañas electorales. En este sentido, es una investigación cualitativa, en la que se describe el uso de las relaciones públicas durante las campañas electorales, tomando como marco de referencia la política electoral en México. Esta metodología implicó la observación sistemática de las campañas electorales durante la elección presidencial del 2018 y del uso de las relaciones públicas durante la misma.

El escrito, se estructura de la siguiente forma. En la primer parte, se habla de lo que son las relaciones públicas. En la segunda parte, se analiza la relación entre las relaciones públicas con la política, en lo general y con la política electoral, en lo particular. En la tercer parte, se describe y analiza el papel que juegan las relaciones públicas en las campañas electorales bajo sistemas político competitivos. En la cuarta parte, se describen y analizan los casos señalados, en la que las relaciones públicas jugaron papeles importantes durante las campañas electorales. Finalmente, se anotan algunas consideraciones finales a manera de conclusión. 


\section{Las relaciones públicas}

Las relaciones humanas y sociales ${ }^{1}$ han estado presentes a lo largo de la historia de la humanidad, desde que el hombre es hombre (Boiry, 2015; Aparecida Ferrari \& Franca, 2011; Arceo, 2004). Sin embargo, las relaciones públicas son un fenómeno relativamente moderno, propio de los sistemas capitalistas, sustentados en la propiedad privada, el libre mercado y la democracia electoral ${ }^{2}$.

Dentro de este tipo de sistemas, las relaciones públicas son entendidas como una función de la administración y el liderazgo de las organizaciones. De esta forma, las relaciones públicas se manifiestan como parte de la convivencia e interacción social, en el ejercicio del liderazgo, en los procesos de intercambio y comercialización de bienes y servicios y, en general, en los procesos de comunicación interpersonal, social y organizacional (PalenciaLefler, 2011).

Históricamente, se considera que las sociedades tribales utilizaron las relaciones públicas por primera vez, con el fin de promover el respeto a la autoridad del jefe y para unir a la tribu ante los peligros que representaban las tribus rivales. Sin embargo, como disciplina, las relaciones públicas se originaron a partir de los procesos de producción de excedentes e intercambio comercial que el naciente capitalismo del siglo XVI trajo consigo en Inglaterra. En la actualidad, las relaciones públicas están presentes en una gran variedad de actos del ser humano y son utilizadas en los procesos de comunicación personal e institucional, con el fin de crear o modificar percepciones, actitudes, creencias y conductas de la gente (públicos específicos) y así influir y ganar aceptación y entendimiento entre las partes involucradas.

Por otro lado, las relaciones públicas han sido conceptualizadas de diferente forma. Para Harlow (1976), las relaciones públicas son una función de la dirección, que ayuda a establecer y mantener líneas de comunicación mutuas, comprensión, aceptación y cooperación entre individuos que integran una organización.

Para la Sociedad de Relaciones Públicas de América (PRSA) las relaciones públicas son "las actividades que ayudan a una organización y a sus públicos a adaptarse mutuamente los unos a los otros." De esta forma, "las relaciones públicas consisten en el proceso estratégico de comunicación que construye relaciones mutuamente beneficiosas entre las organizaciones y sus públicos" (Marketingdirecto.com, 2012).

1 Las relaciones humanas se definen como el conjunto de interacciones entre individuos. Por su naturaleza gregaria, se relacionan y comunican con otros formando grupos para sobrevivir, enfrentar las adversidades y desarrollarse. Por su parte, las relaciones sociales son los vínculos que se establecen entre personas, grupos, familias, clanes, tribus y diferentes sectores sociales, cuya finalidad central es la convivencia y la integración social.

2 Las relaciones públicas están estrechamente ligadas a los sistemas de impronta democrática, sustentados en la libre competencia política y en la existencia de mercados electorales, cuando se forman los públicos (clientes o ciudadanos). La finalidad es realizar relaciones de intercambio y la construcción de consensos sociales. Incluye procesos de influencia e incide en el comportamiento de los ciudadanos, en su papel de consumidores, clientes o electores. 
Según Weinstein, citada por Merca2.0 (2013), las relaciones públicas son “el arte y la ciencia de compartir noticias genuinas, creíbles y relevantes, que mantengan y protejan la aceptación de la marca, el conocimiento, la reputación y las ventas cuando es apropiado. Las relaciones públicas crean conversaciones medibles, basadas en hechos, eventos y actividades concebidas para generar aprobaciones y audiencias de parte de terceros". Para Castillo (2010) las relaciones públicas es la ciencia que se encarga de gestionar las comunicaciones entre una organización y la sociedad con el objetivo de construir, administrar y mantener su imagen positiva.

En este escrito, se llama relaciones públicas a la disciplina encargada de gestionar las relaciones, la comunicación, la imagen, la identidad y la reputación de las personas y las organizaciones con el fin de obtener la simpatía, las preferencias, el consenso, el apoyo y la legitimidad social necesarios para poder lograr los objetivos estratégicos de dichas personas y organizaciones (Black, 2004; Wilcox, Cameron \& Xifra, 2012).

Las relaciones publicas juegan un papel muy importante en el éxito personal y de las organizaciones, de tal manera que se han convertido en una necesidad, ya que ayudan a legitimar a las instituciones y a sus líderes, generan el consenso social necesario dentro en todo sistema democrático y, sobre todo, logran que las empresas aumenten sus ventas, mejoren su marca y alcancen sus objetivos organizacionales. Las relaciones públicas se encuentran presentes en todas las áreas del desarrollo nacional, incluyendo la política y las campañas electorales. A continuación, se describirá la relación y el uso de las relaciones públicas en la política.

\section{Las relaciones públicas y la política}

La política ha sido definida de diferentes maneras. Desde la perspectiva etimológica, la política es la actividad referente a la ciudad o al gobierno de la ciudad. Es el arte de gobernar para conseguir el bien común y la realización de la justicia. Asimismo, es la actividad relacionada con la consecución y el ejercicio del poder en una ciudad. De acuerdo con Weber (1992), la política es la aspiración a participar en el poder o de influir en la distribución del poder entre los distintos Estados o dentro de un mismo Estado, entre los distintos grupos de hombres que lo componen. Quien hace política aspira al poder y genera influencia.

Por su parte, para Schmitt (1991), la política hace referencia el enemigo, a las formas como se derrota y enfrenta a los adversarios. Para Heller (1985), la política es la actividad dirigida a la actuación y organización de la cooperación social en un territorio. Finalmente, para MacIver (1966), la política implica una asociación para mantener el orden social a través de un gobierno que actúa por medio de la ley.

Por su parte, las relaciones públicas han sido conceptualizadas por Harlow (1976) como una función de la dirección que ayuda a establecer y mantener líneas de comunicación mutuas, comprensión, aceptación y cooperación entre individuos y las organizaciones. 
De esta manera, las relaciones públicas y la política encuentran áreas de coincidencia en la comunicación y los vínculos que se dan entre personas u organizaciones, ya que ambas se sustentan en los diferentes nexos y redes que se establecen entre individuos en un espacio y tiempo determinado. Además, tanto la política como las relaciones públicas implican cooperación entre personas, procesos de influencia y disputa por el acceso o conservación del poder en toda sociedad democrática.

Es decir, la política, en su acepción más básica, implica una relación entre dos o más personas y las relaciones implican un nexo político entre personas, ya que toda relación es fundamentalmente política y toda política implica una relación de poder.

Se considera que el primer uso de las relaciones públicas en el campo político en los Estados Unidos de Norteamérica, lo realizó Wilson en 1917 al crear el Comité de Información Pública (Comité Creel), cuyo objetivo central era que los americanos apoyarán la entrada de su nación a la primera guerra mundial. En el caso de México, las relaciones públicas en la política electoral, la impulsaron los primeros candidatos a la presidencia de la república (Vicente Guerrero, Manuel Gómez Pedraza y Anastasio Bustamante), quienes en 1828, trataron de convencer a las legislaturas estatales para que votaran a su favor.

\section{Las relaciones públicas en las campañas electorales}

Las campañas electorales son procesos políticos rutinarios, bajo sistemas de cuño democrático, en la que un candidato, partido o coalición de partidos busca persuadir a los electores para que voten por él y así pueda obtener la mayoría de sufragios en una elección competida con el objetivo de acceder o conservar posiciones de poder político ${ }^{3}$ (Godínez \& Cornejo, 2017).

En el caso de la política electoral, las relaciones públicas se conceptualizan como el conjunto de acciones que se realizan para que un candidato a un puesto de representación pública, partido político o coalición de partidos, se den a conocer entre el electorado, creen una corriente de simpatía y apoyo hacia sí mismos y puedan obtener el mayor número de votos (Mercado, 2002).

Las relaciones públicas implican la relación, persuasión e incorporación de diferentes liderazgos a las campañas electorales. Estos liderazgos pueden ser de carácter no solo político, sino también social, económico, religioso o líderes de opinión pública. Estos liderazgos, ejercen un gran poder de influencia sobre amplios grupos sociales, en su papel de electores, por lo que incorporar liderazgos a las campañas suele ser una estrategia de relaciones públicas muy socorrida por partidos políticos y candidatos a un puesto de elección popular.

3 Las campañas electorales son ejercicios políticos rutinarios, bajo sistemas democráticos, que se presentan generalmente en el espacio público y que incluye la participación de una cantidad determinada de electores, quien decide el carácter de la representación pública. Su voto implica, de cierta manera, decidir sobre la continuidad o el cambio. 
Las relaciones públicas se hacen presentes en todo el proceso de comunicación política durante una campaña electoral a través de las redes sociales, las páginas web, los blogs, las video conferencias, los mítines y reuniones digitales, así como las diferentes acciones comunicativas que se den a través de los diferentes medios, en los que se usan las nuevas tecnologías de la información y las comunicaciones.

Por medio de las relaciones públicas, los candidatos a un puesto de representación popular establecen vínculos y comunicación directa e indirecta con diferentes ciudadanos, líderes, dirigentes de organizaciones políticas, económicas y sociales, así como con los diferentes sectores sociales. De igual forma, los candidatos se vinculan con sus aliados políticos e incluso, en algunas ocasiones establecen relación con sus adversarios.

Las relaciones públicas implican un proceso eminentemente de comunicación, el cual, durante las campañas electorales, se convierten en un eje estratégico de la acción política, que ayudan a conseguir una mayoría de votos. Esto se convierte, la mayoría de las veces, en el objetivo central de dichos ejercicios proselitistas.

Las relaciones públicas también están presentes en las diferentes actividades de promoción del voto, en las visitas domiciliarias puerta a puerta, la promoción del voto en cruceros y avenidas de las ciudades y pueblos, y en los distintos eventos de contacto directo con los electores. Es decir, las campañas electorales se sustentan en las relaciones que se establecen en el frente territorial (contacto directo con los electores), el frente mediático (relaciones a través de la televisión y radio principalmente) y el frente digital (a través de las redes sociales).

Toda campaña electoral implica, a su vez, un proceso de investigación, comunicación, organización, movilización, cuidado y defensa del voto en la que las relaciones públicas también están presentes y juegan un papel muy importante. Por ejemplo, en el proceso de investigación sobre las características distintivas del mercado electoral, así como del contexto y la coyuntura política, las relaciones públicas están presentes desde la gestión de la idea de la investigación desde la pre-candidatura, pasando por el diseño del instrumento de investigación y hasta la interpretación y comunicación de resultados de los estudios sobre conocimiento e imagen de los candidatos, así como de las preferencias electorales de los ciudadanos.

En el proceso de comunicación política, las relaciones públicas están presentes durante las campañas en las diferentes acciones que se emprenden desde la edición y transmisión de los spots publicitarios, la relación con los diferentes medios de comunicación, las campañas de promoción en redes sociales, los eventos y concentraciones públicas, la publicidad callejera y la impresión de los diferentes objetos utilitarios, entre otros. Es decir, las relaciones públicas son ejes estratégicos centrales durante las campañas electorales, jugando un papel protagónico durante las elecciones.

En el proceso de movilización, las relaciones públicas también juegan un papel muy importante para asegurar que los electores acudan a emitir su sufragio, generando las 
condiciones y dotando de la información necesaria para que los ciudadanos voten y participen en los procesos electorales.

En el proceso de organización, las relaciones públicas también están presentes durante las campañas electorales desde el establecimiento del organigrama de campaña, la organización de reuniones de planeación y evaluación de las actividades de campaña, hasta en la construcción o relación con estructuras políticas, económicas y sociales en apoyo a la campaña. La organización implica la arquitectura política o el diseño organizacional de la campaña en la que las relaciones públicas están presentes en la conformación del equipo de campaña, la relación con los consultores y asesores, así como en la organización de las diferentes estructuras electorales que se conforman para la promoción del voto.

En el proceso de cuidado y defensa del voto, también las relaciones públicas están presentes en la conformación de las estructuras electorales que cuidan y defienden el voto antes, durante y después de la jornada electoral. Las relaciones públicas también son parte importante durante la etapa de reclutamiento y capacitación de los representantes de casilla y representantes generales de los partidos y candidatos, así como durante todo el proceso de lo contencioso electoral. Es decir, las relaciones públicas juegan un papel estratégico durante toda campaña electoral, de tal forma que no puede pensarse un ejercicio político de esta naturaleza, sin la competencia y la intervención de las relaciones públicas y la comunicación, como parte del proceso directivo.

\section{Resultados: las relaciones públicas como procesos de intercambio}

Las relaciones públicas también se pueden conceptualizar como un proceso de intercambio en el que los candidatos a un puesto de representación popular y sus equipos de campaña intercambian desde mensajes, propuestas e ideas con los ciudadanos hasta proyectos alternativos de nación. Por su parte, los ciudadanos plantean ante los políticos sus opiniones, demandas, necesidades, deseos, aspiraciones, protestas y quejas sobre los aspectos que conciernen a su comunidad, el sistema electoral y su gobierno.

En este sentido, una campaña también es un proceso de intercambio entre políticos y ciudadanos. En este proceso, las relaciones públicas se hacen presentes durante las campañas en la construcción de imagen, en la investigación de opinión púbica, en la relación con los medios de comunicación, en los diferentes eventos que se organizan para promover y publicitar a los candidatos, a los partidos políticos y a sus plataformas programáticas. También, se encuentran presente a través de la logística y el protocolo que se utiliza en los diferentes eventos que se organizan como parte de la campaña. A continuación, se explican, a más detalle, cada uno de ellas.

\subsection{Imagen y reputación}

La imagen es la percepción que los demás tienen o se forman de otra persona, misma que se establece como parte de una relación social que se da en un tiempo y espacio determinado. 
Es decir, la imagen es percepción y las relaciones públicas ayudan a construir, reconstruir y mejorar la imagen de los candidatos que participan en un proceso electoral, de los partidos políticos y de las propias campañas electorales. De hecho, una campaña se convierte en un ejercicio para moldear la percepción de los electores y para que estos voten a favor de su persona, su partido coalición de partidos políticos o por su plataforma programática. De esta forma, las relaciones públicas juegan un papel importante durante las campañas electorales, ya que los electores generalmente deciden su voto a partir de sus percepciones.

En una campaña electoral, son importantes tres tipos de imagen: la del candidato, la del partido que lo postula y la del equipo de campaña. En primer lugar, la imagen del candidato es la percepción que los electores tienen sobre su persona, sus cualidades físicas e intelectuales, sus ideas, sus propuestas, sus capacidades, su honorabilidad y liderazgo, mismas que se han formado a través de los años por la información que han obtenido de forma directa o indirecta.

La imagen del partido o coalición de partidos, que postulan a un candidato, se refiere a la percepción que los votantes tienen de los partidos políticos o coaliciones electorales que se forman en la campaña y que motivan o desmotivan a los votantes a asistir a las urnas y a depositar su voto a favor de alguno de los partidos contendientes. Por último, la imagen del equipo de campaña se forma a partir de la relación que se establece entre los integrantes del equipo de campaña con los electores en la realización de actividades de promoción del voto y de las múltiples actividades de carácter político que se realizan durante el periodo electoral.

\subsection{Gestión de crisis}

Las crisis están presentes en todas las campañas electorales. Estas pueden manifestarse en forma de poco o nulo apoyo popular, escándalos, descredito, divisiones, rupturas, renuncias o ataques de los adversarios, entre otras maneras. Su efecto puede ser desde daños menores hasta daños mayores y devastadores para las campañas electorales. Gestionar la crisis implica encontrar formas creativas y funcionales para salir de ella y acciones que permitan aminorar sus efectos perniciosos o dañinos. Hablar, escuchar, actuar con rapidez, honestidad y cercanía son parte de un protocolo de actuación que resulta sencillo y funcional (Losada, 2018).

La gestión de la crisis implica un diagnóstico sobre las causas de la misma, las consecuencias que está generando y las formas o estrategias para tratar de salir de ellas. También implica el proceso mismo de intervención organizacional, así como el proceso de evaluación que posibilite obtener aprendizajes importantes para el manejo de la crisis.

\subsection{Relación con medios de comunicación}

Los medios de comunicación son actores muy importantes durante las campañas electorales. Los medios inciden en la formación de opinión pública y en moldear las percepciones sociales sobre la política y las elecciones. De hecho, se puede decir que los medios son capaces de 
hacer que las candidaturas sean exitosas o de destruirlas, según sea o corresponda al interés y deseo de sus propietarios y representantes.

En este sentido, toda campaña debe buscar tener una buena relación con los medios de comunicación, utilizando la oportunidad para poder comunicarse con los electores a través de su conducto, tratando de incidir positivamente en la opinión pública y en la formación de percepciones sociales sobre la campaña, los candidatos y la elección.

Las relaciones públicas deben estar orientados a fomentar un acercamiento del candidato y la campaña con los medios de comunicación, que le permita la cobertura mediática de los principales eventos, así como dotar de visibilidad social al candidato y a sus principales propuestas y mensajes de campaña.

\subsection{Eventos, logística y protocolo}

Las relaciones públicas están presentes en la organización de eventos, en su logística y en las actividades de protocolo de las campañas electorales. Se puede decir, que una campaña se compone por un conjunto de eventos que organizan los partidos políticos y candidatos para tratar de persuadir a los electores, durante el tiempo que señala la ley o reglamento que norma las elecciones. De hecho, los eventos son herramientas de comunicación que también transmiten mensajes (Cuadrado \& Rodríguez, 2015).

Si los eventos están bien organizados, se cuida con esmero la logística de la campaña y esta observa los protocolos adecuados, entonces las posibilidades de éxito de la campaña aumentan. Por el contrario, si la organización de eventos durante la campaña electoral es deficiente, se presentan diferentes problemas en la logística y el protocolo de los eventos, entonces las posibilidades de éxito de la campaña electoral disminuyen.

El éxito o fracaso de la campaña está en relación directa con el número de eventos que se impulsan para persuadir y movilizar a las urnas a los electores, así como con la cantidad de electores que participen en ellos y la calidad de los mismos, su logística y su protocolo. Esto implica la presencia de las relaciones públicas como eje articulador de la campaña electoral.

\subsection{Cabildeo o lobbing}

El lobbing puede definirse como las acciones de cabildeo que se llevan a cabo por un grupo de personas para tratar de incidir en una decisión de una persona, institución o grupo, tratando de que esta decisión sea favorable a los intereses de quienes impulsan este tipo de acciones. Este tipo de cabildeo lo realizan generalmente los publi-relacionistas, quienes tratan de incidir en las decisiones de los demás.

En este sentido, las relaciones públicas implican el impulso de ciertas acciones de cabildeo (también denominada lobbing) con el fin de poder incidir en la decisión de los grupos de poder y en los diferentes actores que juegan roles protagónicos en los procesos electorales. 
De esta manera, las relaciones públicas posibilitan el apoyo financiero de personas o grupos económicos hacia determinados partidos o candidatos a un puesto de representación popular, el respaldo de ciertas personalidades en apoyo a la campaña y el involucramiento de los ciudadanos con una determinada opción política.

Las campañas electorales se ganan con la participación y el involucramiento no solo de los electores, sino también de grupos de poder y de diferentes liderazgos que existen en todos los sistemas democráticos modernos. Para lograr su involucramiento, se usan diferentes acciones de gestión o lobbing, que posibilite ampliar los apoyos necesarios para ganar elecciones.

\section{A manera de conclusión}

Las relaciones públicas se encuentran presentes en los procesos centrales de las campañas electorales, bajo sistemas políticos de cuño democrático. En esencia, impulsar una campaña electoral implica un ejercicio de relaciones públicas con el objetivo de conocer a los electores y de diseñar diferentes estrategias para conseguir su voto y así evitar que los adversarios logren arribar a posiciones de representación pública.

La política es un ejercicio de contacto que se ejerce a través de una serie de relaciones e interrelaciones con el fin de alcanzar ciertos objetivos previamente establecidos. El contacto implica relaciones. En el caso de la política electoral y las campañas, el objetivo central es obtener el mayor número de votos, el cual puede lograrse a través del uso estratégico de las relaciones públicas. De esta forma, una campaña electoral implica un ejercicio inteligente, oportuno y creativo de relaciones públicas, con el fin de conservar o acceder al poder político.

A través de un buen plan de relaciones públicas, es posible conocer la opinión pública, moldear las percepciones sociales, cambiar sus actitudes y decisiones, formar una mejor imagen de los candidatos y partidos políticos, comunicarse con los electores, organizarlos y poder movilizarlos el día de las elecciones. En una campaña electoral, todo se reduce a un adecuado manejo de las relaciones públicas.

Así, el éxito o fracaso de una campaña electoral dependerá de la capacidad que se tenga como individuos o como organizaciones para lograr un manejo creativo, oportuno e inteligente de las relaciones públicas. Puesto de otra manera, se puede decir que a mejor uso de las relaciones públicas corresponde una mayor posibilidad de ganar un mayor número de votos y así obtener las posiciones de poder político que implican toda campaña electoral bajo un sistema democrático.

Las relaciones públicas están en proceso permanente de evolución y desarrollo. A partir de la revolución tecnológica global, predominan en las relaciones públicas los medios digitales, la reputación online, el uso de la inteligencia artificial (big data), la identidad e imagen digital, los nuevos influencers a través de las redes sociales, el storydoing, la 
consultoría política, la comunicación directa e inmediata en un entorno más participativo e interactivo. En este sentido, las relaciones públicas se están modernizando, impactando y transformando también la forma como se impulsan y gestionan las campañas electorales en todo el orbe.

\section{Fuentes consultadas}

Álvarez, A. (2011). Medición y evaluación en la comunicación. Málaga: Universidad de Málaga.

Aparecida Ferrari, M. \& Franca, F. (2011). Relaciones Públicas: Naturaleza, función y gestión en las organizaciones contemporáneas. Buenos Aires: La Crujía.

Arceo, J. (2004). Las Relaciones Públicas en España. Madrid: McGraw-Hill.

Black, S. (2004). ABC de las Relaciones Públicas. Madrid: Gestión 2000.

Boiry, P. (2015). Relaciones públicas o la estrategia de la confianza. Madrid: Gestión 2000.

Castillo, E. (2010). Introducción a las Relaciones Públicas. Málaga: Universidad de Málaga.

Colombo, D. (2004). Sea su propio jefe de prensa. México, DF: Norma.

Cuadrado, C. \& Rodríguez, R. (2015). El ABC de la organización de eventos. México, DF: FC Editorial.

Godínez, J. \& Cornejo, J. (2017). La nueva guerra: el marketing político. Te digo cómo ganar. Guadalajara: Universidad de Guadalajara.

Harlow, R. (1976). Building a Public Relations. Nueva York: Holt, Rinehart \& Winston.

Heller, H. (1985). Escritos políticos. Madrid. Alianza Universidad.

Losada, J. (2018). (No)crisis. La comunicación de crisis en un mundo conectado. Barcelona: Editorial UOC.

MacIver, R. (1966). Teoría del gobierno. Madrid: Tecnos.

Mares, E. (2011). El vocero ante los medios. Buenos Aires: Deuro.com.

Marketingdirecto.com (5 de marzo de 2012). Las relaciones públicas estrenan nueva definición. Recuperado de https://www.marketingdirecto.com/marketing-general/ tendencias/las-relaciones-publicas-estrenan-nueva-definicion 
Merca2.0 (19 de diciembre de 2013). ¿Qué son las relaciones públicas? 4 definiciones. Recuperado de https://www.merca20.com/que-son-las-relaciones-publicas-4definiciones/

Mercado, S. (2002). Las RR. PP. aplicadas. Un camino hacia la productividad. México, DF: Thompson Learning.

Molinari, G. (2008). El ceremonial institucional y de los negocios. Buenos Aires: UADE.

Palencia-Lefler, M. (2011). 90 técnicas de comunicación y relaciones públicas. Barcelona: Bresca Editorial.

Schmitt, C. (1991). El concepto de lo político. Madrid: Alianza Editorial.

Valdez, A. (2018). Liderazgo y gerencia de campañas electorales. Maracaibo: Universidad del Zulia.

Weber, M. (1992). El cientifico y el político. Madrid: Alianza Editorial.

Wilcox, D., Cameron, G. \& Xifra, J. (2012). Relaciones Públicas. Estrategias y tácticas. Madrid: Pearson Educación. 